\title{
Dynamic Matrix Control DMC using the Tuning Procedure based on First Order Plus Dead Time for Infant-Incubator
}

\author{
J. El Hadj Ali ${ }^{1}$, E. Feki, A. Mami ${ }^{2}$ \\ University of Tunis El Manar, Faculty of Science \\ UR17ES11 LAPER, 2092 Tunis, Tunisia
}

\begin{abstract}
The concept of Model Predictive Control (MPC) is considered as one of the most important controlling strategies. It is used in several fields, such as petrochemical, oil refinery, fertilizer and chemical plants. It is also well spread among the clinicians and in the biomedical fields. In this context, our paper aims to investigate the thermal conditions inside the infant incubator for premature babies. In this study, we propose the Dynamic Matrix Control (DMC) as a control strategy. The most particularity of this strategy is applicable to the Multi-input Multi-output (MIMO) systems. It aims to compare different coupled transfer functions achieved by two identification methods in previous work. Also, a simulation of the air temperature and humidity is achieved inside the unit care. In this work, we focus on the tuning controlling parameters because it is considered as a key step in the successful performance of (DMC). Then, to obtain the (DMC), we have used an analytic tool, which is the Process Reaction Curve (PRC), for higher order transfers function because it needs a lot of work for this purpose. It should be approximated as a low order transfer function with time delay, which is achieved by using the First Order Plus Dead Time (FOPDT) of processing models. Finally, the result of the comparison of the infant-incubator is provided to show an optimal and good performance of the thermal behavior of our propos methodology and prove that a good identification ensures a better performance.
\end{abstract}

Keywords-Infant-incubator; DMC; MPC; higher-order; FOPDT; PRC and MIMO

\section{INTRODUCTION}

It is well-known that the period from 1970 to 1980 witnessed the appearance of the first generation of Model Predictive Control (MPC). One of the most popular MPC technologies called Dynamic Matrix Control (DMC) was developed by a crew of engineers from the Shell Oil company lead up by Culter and Remarker [1, 2]. Also, in 1980, Prett and Gillette [3] presented an application of DMC. Since the existence of the first generation of MPC, this latter has a large effect on the image of the industrial applications.

The DMC is a linear control technique which uses the First Order Plus Dead Time (FOPDT) model. The advantage of this method has already been established and has been foun to work correctly for a long period of time DMC is used in the majority of industrial applications, where its biggest particularity lies in the MIMO systems. Besides, the use of the model response to a unit step change is needed to predict the future response of the dependent parameters and formulates a sequence of control actions for all independent variables. The series of control action is chosen to minimize the error of the process over the time horizon. Also, it is applicable to multivariable processes as long as they are asymptotically stable and without integrators (Prada, Serrano, \& Vega, A comparative study of GPC and DMC controllers, 1994) [4]. DMC is used in the implementation of Single-input Singleoutput (SISO) and MIMO systems, but in this study, focuses only on the multivariable case, particularly two decoupled inputs and two outputs (the temperature and the humidity inside the unit care of the infant incubator).

Over the years the technology witnessed a development in all fields, especially in the biomedical field. Until today, the infant-incubator for the preterm babies is considered as one of the important issues is in the neonatal field The incubators have been designed to achieve a helpful and appropriate hygrothermal environment for the newborn babies. In this context, a neonatal incubator requires agreeable conditions to establish a good range of temperature and humidity also a minimum waste energy. In general the closed incubator structure is depicted with four walls made of one layer of Plexiglas Fig. 1. In addition, all infant incubators perform in the same manner. The fan is used to circulate warm air over a heating element and a water container through two small ports. Also, the majority of the incubator system has a passive humidification system. But in this case, the heater is replaced by a dimmer to permit external control as it is an infinitely variable control.

The essential objective of this study is to develop a mathematical model of the infant incubator and obtain a good tuning of the control strategy to ameliorate the performance of the control inside this device for coupling temperature and humidity and prove that the identification and the tuning play a radical way in the final response.

The outlines of this paper start with a brief introduction. Then, Section 2 presents the related works of the infant incubators over the past years. The dynamic matrix control modeling for MIMO case is described in Section 3. As for Section 4, it highlights the validation of the tuning strategy of the two methods and discusses the simulation results. Then, Section 5 is dedicated to a comparative study of both methods. Finally, the concluding remarks and future works are presented in Section 6. 


\section{RELATED WORKS}

Over the past few decades, the problem of preterm infants and congenital patients has been addressed as a very important issue. For this reason, many studies in the literature have focused on controlling the relative temperature and humidity of an infant incubator with several strategies of control due to demands to improve the performance of the intensive care unit. Despite all the research and developments in this context, there is still little focus on the incubators controller with the predictive controls, which is the interest of this study.

The predictive control strategy was first brought into use in 2006, within the study of Gustavo H. C. Oliveira which is based on the identification of the Laguerre function [5]. Then, the same researchers proposed a hybrid predictive control for mixed logical and dynamical models [6].

In 2010, the authors of this paper developed a robust predictive control strategy for multivariable systems with multiple delays and input constrains [7].

In [8], the same authors developed an application of Indirect Adaptive Generalized Predictive Control compared with ON-OFF and PID controller. In the same year, they designed a new active system which is able to generate the control of humidity and carried out a comparative study between GA-PID and GA-MPC [9].

In 2014, the authors developed a theoretical model for the thermal behavior (air temperature and relative humidity) which was controlled by decoupling the generalized predictive controller (DGPC) in order to achieve optimal thermal conditions [10].

In [11], in this study, they have designed the Simulink model using Generalized Predictive Control (GPC) as compared with PID control with and without newborn inside the infant incubator.

\section{DYNAMIC MATRIX CONTROL MATHEMATICAL MODELING}

\section{A. Dynamic Matrix Control Mathematical Modeling}

The Since the appearance of DMC in the literature, researches have detailed the derivation of the MIMO-DMC control law [12, 13], Prett and Gracia [14], it can be considered as an extension of the SISO case dealt with in the previous works $[1,2,17]$. The former researchers were deeply reviewing the mathematical formulation and the tuning parameters of DMC [15]. For the sake of this paper, only a short study and a recap of DMC will be presented below.

In our case, considering a system of two inputs, two outputs TITO $(2 \times 2)$, we then obtain the output from the step response coefficients, as follows [16, 17]:

Where $\mathrm{u}_{\mathrm{i}}(\mathrm{k})$ and $\Delta \mathrm{u}_{\mathrm{i}}(\mathrm{k})$ are considered as the $\mathrm{i}^{\text {th }}$ input besides its variation in sample time $\mathrm{k}$ as $\mathrm{u}_{\mathrm{i}}(\mathrm{k})-\mathrm{u}_{\mathrm{i}}(\mathrm{k}-1)$. In addition, the step response coefficients at sample time $i$ are $\mathrm{a}_{\mathrm{i}}$, $b_{i}, c_{i}$ and $d_{i}$. Also, the sample time $\mathrm{N}$ at each step response reaches its steady state.

$$
\left\{\begin{aligned}
y_{1}(k)= & \sum_{i=1}^{N} a_{i} \Delta u_{1}(k-i)+a_{N+1} u_{1}(k-N-1) \\
& +\sum_{i=1}^{N} b_{i} \Delta u_{2}(k-i)+b_{N+1} u_{2}(k-N-1)+d_{1}(k) \\
y_{2}(k)= & \sum_{i=1}^{N} c_{i} \Delta u_{1}(k-i)+c_{N+1} u_{1}(k-N-1) \\
& +\sum_{i=1}^{N} d_{i} \Delta u_{2}(k-i)+d_{N+1} u_{2}(k-N-1)+d_{2}(k)
\end{aligned}\right.
$$

The appearance of any difference or change between the manipulated output (measuring system) and the predicted output among the above model is presented by equation (2):

$$
\left\{\begin{array}{l}
d_{1}(k)=y_{1}^{\text {meas }}(k)-y_{1}^{\text {model }}(k) \\
d_{2}(k)=y_{2}^{\text {meas }}(k)-y_{2}^{\text {model }}(k)
\end{array}\right.
$$

Where $y^{\text {meas }}$ and $y^{\text {model }}$ are the measured output and the output of the model respectively. These errors refer to the mismatch between the model/system and external disturbances (noises). In addition, $P$ is known as the future predictions of the system output established on a control horizon $M$. They are described on the following matrix-vector form which yields the following:

$S_{f}=\left[\begin{array}{cccc|cccc}a_{1} & 0 & \ldots & 0 & b_{1} & 0 & \ldots & 0 \\ a_{2} & a_{1} & \ldots & 0 & b_{2} & b_{1} & \ldots & 0 \\ \vdots & \vdots & \vdots & \vdots & \vdots & \vdots & \vdots & \vdots \\ a_{M} & a_{M-1} & \ldots & a_{1} & b_{M} & b_{M-1} & \ldots & b_{1} \\ \vdots & \vdots & \vdots & \vdots & \vdots & \vdots & \vdots & \vdots \\ a_{p} & a_{p-1} & & a_{p-M+1} & b_{p} & b_{p-1} & \ldots & b_{p-M+1} \\ \hline c_{1} & 0 & \ldots & 0 & d_{1} & 0 & \ldots & 0 \\ c_{2} & c_{1} & \ldots & 0 & d_{1} & d_{1} & \ldots & 0 \\ \vdots & \vdots & \vdots & \vdots & \vdots & \vdots & \vdots & \vdots \\ c_{M} & c_{M-1} & \ldots & c_{1} & d_{1} & d_{1} & \ldots & d_{1} \\ \vdots & \vdots & \vdots & \vdots & \vdots & \vdots & \vdots & \vdots \\ c_{p} & c_{p-1} & \ldots & c_{p+M-1} & d_{p} & d_{p-1} & \ldots & d_{P-M+1}\end{array}\right]$

For the case of the multi variable (TITO) the Dynamic matrix process is achieved by the coefficients of the four step response models from the plant. It is represented by the dimension as $(2 P \times 2 M)$.

The move suppression weight which is known as $\gamma$ is used to form the diagonal matrix which is represented by $\Gamma$ and given by:

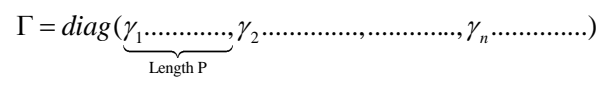

Where $\mathrm{n}$ is the number of the manipulated variables and $(\mathrm{nP} \times \mathrm{nP})$ is the dimension of the move suppression matrix. The diagonal matrix is formed by using the control variable weights $(\lambda)$ which is depicted by the following equation:

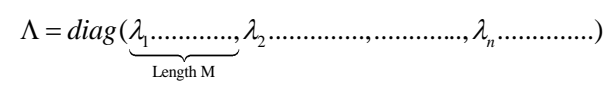

The dimension of the control variable weight matrix is $(n M \times n M)$. 
The previous expression equation (5) can be rewritten with a matrix vector as follows equation (6):

$$
y^{\text {lin }}=S_{f} \Delta u+y^{\text {past }}+d
$$

Where $\mathrm{P}$ and $\mathrm{M}$ are the prediction and control (moving) horizons, respectively; and $\mathrm{y}^{\text {past }}$ indicates the effects of the past inputs on the predicted outputs in the future. Also, the matrix A, which is named a dynamic matrix of the process, depends on the step response coefficients.

Likewise, $\Delta \mathrm{u}$ which is the control moves is calculated according to the solution to the optimization problem of the Two input Two output, in this case, such that:

$$
\operatorname{Min}_{\Delta u}\left(y^{s p}-y^{l i n}\right)^{T} \Gamma^{T} \Gamma\left(y^{s p}-y^{l i n}\right)+\Delta u^{T} \Lambda^{T} \Lambda \Delta u
$$

\section{Where}

$y^{s p}:$ is the desired output trajectory,

$\Gamma$ : is the weighting matrices on the prediction error, and,

$\Lambda:$ is the control effort.

Under the unconstraint minimization, the optimal input is determined as follows:

$$
\Delta u=\underbrace{\left(S_{f}{ }^{T} \Gamma^{T} \Gamma S_{f}+\Lambda^{T} \Lambda\right)^{-1} S_{f}{ }^{T} \Gamma^{T} \Gamma}_{K} \underbrace{\left(y^{s p}-y^{\text {past }}-d\right)}_{\text {Error vector } \overline{\mathrm{e}}}
$$

Generally, the first line of the $\Delta \mathrm{u}$ is applied to the system. This procedure is performed in each sampling interval.

\section{B. Role of the Tuning Parameters of Multi-Input Multi- Output of Dynamic Matrix Control}

The design of the DMC controller enables the adjustment of the prediction horizon and control horizon, which presents an optimal control action for the process. For that reason, the tuning parameters of the DMC along with the control horizon $\mathrm{M}$, prediction horizon $\mathrm{P}$ and sample time $\mathrm{T}$ can provide a superior performance of controlling the humidity and temperature in the unit care of newborn babies. In this paper, user-friendly tuning strategy is developed and defined based on an algorithm that will calculate the above-mentioned parameters for an unconstrained MIMO system [18, 19]. Dougherty and Cooper (2003) [20] proposed some guidelines in order to use the MIMO-DMC. These guidelines are summarized in the next 7 steps $[21,22]$ :

\section{- Step1}

Approximate the process dynamics of the controller output to measure the process variable pairs of the integrating sub processes with FOPDT models, as follows:

$$
\frac{y_{j}(s)}{u_{i}(s)}=\frac{K_{p_{i j}}^{*} e^{-\theta_{p_{i j}} s}}{\tau_{p_{i j}} s+1} \text { for }(\mathrm{i}=1,2, \ldots, \mathrm{S} ; \mathrm{j}=1,2, \ldots, \mathrm{R})
$$

\section{- Step2}

Select the sample time as close as possible to

$$
\boldsymbol{T}=\mathbf{0 . 1} \tau_{i j} \quad \text { or } \quad \boldsymbol{T}=\mathbf{0 . 5} \boldsymbol{\theta}_{i j},
$$

Whichever is smaller $(i=1,2, \ldots, S ; j=1,2, \ldots, R)$

\section{- Step3}

Compute the prediction horizon $\mathrm{P}$ and the model horizon, $\mathrm{N}$, as the process settling time in samples (rounded to the next integer), such that:

$$
P=N=\operatorname{Max}\left(\frac{5 \tau_{i j}}{T}+k_{i j}\right)
$$

where

$$
k_{i j}=\left(\frac{\theta_{i j}}{T}+1\right)_{(i=1,2, \ldots, S ; j=1,2, \ldots, R)}
$$

\section{- Step4}

Select the control horizon $\mathrm{M}$ as an integer (usually between 1 to 6 ).

\section{- Step5}

Select the controlled variable weights $\gamma_{\mathrm{J}}^{2}$ to scale the measurements to similar magnitudes.

\section{- Step6} follows:

Compute the move suppression coefficients, $\lambda_{J}^{2}$, as

$$
\lambda_{i}^{2}=\frac{M}{500} \sum_{j=1}^{R}\left[\gamma_{j}^{2} K_{i j}^{2}\left(P-k_{i j}-\frac{3}{2} \frac{\tau_{i j}}{T}+2-\frac{(M-1)}{2}\right)\right]
$$

\section{- Step7}

Implement DMC using the traditional step response matrix of the actual process and the initial values of the parameters computed in steps 1 to 6 .

\section{Tuning Strategy Validation for Multivariable PROCESS AND DISCUSSION}

Fig. 1 presents the real photo of the infant incubator, the isolette $®$ Dräeger $8000 \mathrm{C}$ which is used in this experiment. In this system, the set point is defined as $37^{\circ} \mathrm{C}$ and the input energy varies between $0-100 \%$, i.e. equivalent to 0 and 400 Watt. An ultrasonic nebulizer, which is an instrument for converting a liquid into a fine spray, is also used. As for the humidifier power, it varies between 0-150 watts. This system is able to increase the humidity by $80 \%$ [12].

\section{A. Case 1: Dynamic Matric Control for Ident Toolbox}

The tuning strategy is provided and validated in this paper to control the temperature and the humidity of the infantincubator. Besides the transfer function of this process, it is also extracted from previous works [23]. It is a system of $(2 \times 2)$ of the coupled system and third order system, as illustrated in the Fig. 2. 


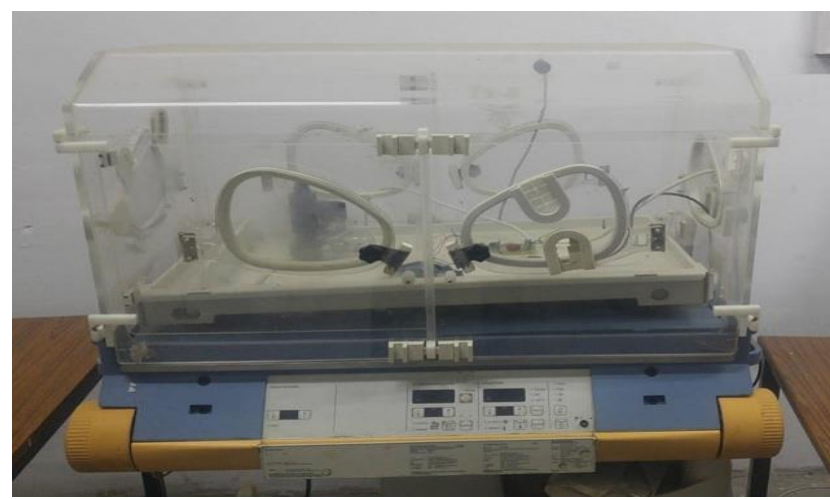

Fig. 1. The Real Photo of the Infant Incubator Dräeger 8000 C.

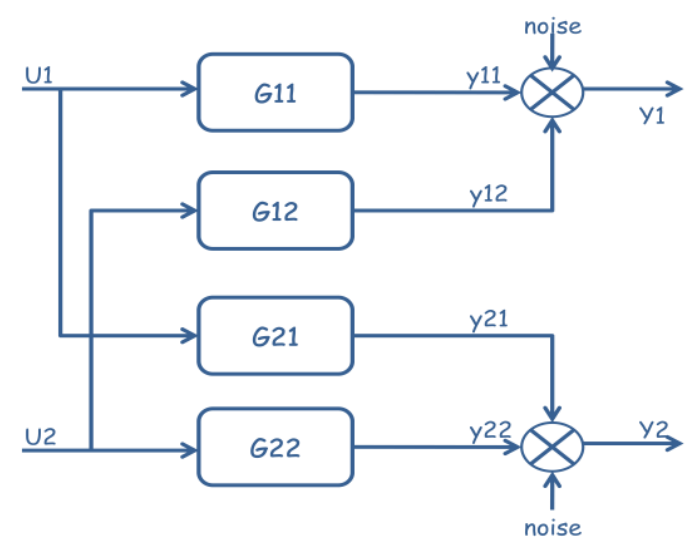

Fig. 2. Two-Input Two-Output Processes.

The transfer function matrix can be written with a sample time $\mathrm{Ts}=20$, as follows:

$G(z)=\left[\begin{array}{ll}G_{11}(z) & G_{12}(z) \\ G_{11}(z) & G_{22}(z)\end{array}\right]=\left[\begin{array}{lr}z^{-d 11} \frac{B_{11}\left(z^{-1}\right)}{A_{11}\left(z^{-1}\right)} & z^{-d 12} \frac{B_{12}\left(z^{-1}\right)}{A_{12}\left(z^{-1}\right)} \\ z^{-d 21} \frac{B_{21}\left(z^{-1}\right)}{A_{21}\left(z^{-1}\right)} & z^{-d 22} \frac{B_{22}\left(z^{-1}\right)}{A_{22}\left(z^{-1}\right)}\end{array}\right]$

Each transfer function of the system is detailed in the following expression:

$$
\left\{\begin{array}{l}
G_{11}(z)=z^{-d_{11}} \frac{B_{11}(z)}{A_{11}(z)}=z^{-10} \frac{0.0005875 z^{-1}+0.0005941 z^{-2}}{1-0.3464 z^{-1}+0.6463 z^{-2}} \\
G_{12}(z)=z^{-d_{12}} \frac{B_{12}(z)}{A_{12}(z)}=0 \\
G_{21}(z)=z^{-d_{21}} \frac{B_{21}(z)}{A_{21}(z)}=z^{-32} \frac{0.000137 z^{-1}-4.193 e-05 z^{-2}}{1-0.2832 z^{-1}+0.7133 z^{-2}} \\
G_{22}(z)=z^{-d_{22}} \frac{B_{22}(z)}{A_{22}(z)}=z^{-3} \frac{0.00203 z^{-1}-0.00088 z^{-2}}{1-0.5091 z^{-1}+0.4262 z^{-2}}
\end{array}\right.
$$

Also, all these functions can be rewritten as a continuous form and it is obvious that the model order is increased to handle real negative poles, as illustrated in equation (11):

$$
\left\{\begin{array}{l}
G_{11}(s)=\exp (-200 * \mathrm{~s}) \frac{3.423 \mathrm{e}-05 \mathrm{~s}^{2}-4.204 \mathrm{e}-07 \mathrm{~s}+9.026 \mathrm{e}-07}{\mathrm{~s}^{3}+0.04343 \mathrm{~s}^{2}+0.02515 \mathrm{~s}+5.577 \mathrm{e}-06} \\
G_{12}(s)=0 \\
G_{21}(s)=\exp (-640 * \mathrm{~s}) \frac{3.569 \mathrm{e}-06 \mathrm{~s}^{2}+1.281 \mathrm{e}-06 \mathrm{~s}+6.924 \mathrm{e}-08}{\mathrm{~s}^{3}+0.03368 \mathrm{~s}^{2}+0.02496 \mathrm{~s}+2.542 \mathrm{e}-06} \\
G_{22}(s)=\exp (-60 * \mathrm{~s}) \frac{0.0001034 \mathrm{~s}^{2}+8.64 \mathrm{e}-06 \mathrm{~s}+2.707 \mathrm{e}-06}{\mathrm{~s}^{3}+0.083 \mathrm{~s}^{2}+0.02649 \mathrm{~s}+6.019 \mathrm{e}-05}
\end{array}\right.
$$

The First Order Plus Dead Time (FOPDT) models are used to represent the behavior of numerous processes for the reason that some responses of such models like step or pulse inputs can propose an interesting and excellent approximation for the actual responses of many systems and sub-systems in many applications. Anyhow, the theoretical development of many system identification algorithms is available in the literature $[25,26]$.

Hence, this system is modeled according the Process Reaction Curve known as (PRC). It is identified by performing in an open loop step test of the process and finding model parameters for the initial step. A typical process reaction curve is generated using the following method [2426]:

1) Find $\Delta y$ from step response.

2) Find $\Delta \mathrm{u}$ from step response.

3) Calculate $\mathrm{k}_{\mathrm{p}}=\frac{\Delta \mathrm{y}}{\Delta \mathrm{u}}$.

4) Find the apparent dead time $\theta_{\mathrm{p}}$, from step response.

5) Find $(0.632 \times \Delta y)$ from step response.

6) Find $t_{0.632}$ for $y\left(t_{0.632}\right)=0.632 \times \Delta y$ from step response.

7) Calculate $\tau_{\mathrm{p}}=\mathrm{t}_{0.632}-\theta_{\mathrm{p}}$.

As a result, of this method, it is obvious that the process reaction curve obtained from the real time model and its approximation is as shown below.

Table I below summarizes the values of each parameter which are taken from the fitting curve and computed by the above method of approximation FOPDTs whose transfer function is given by $\mathrm{G}_{\mathrm{ij}}^{\text {approx }}$.

TABLE I. APPROXIMATION COMPUTED VALUES AS FOPDT

\begin{tabular}{|l|l|l|l|l|l|l|l|}
\hline & $\Delta \mathbf{y}$ & $\boldsymbol{\Delta u}$ & $\mathbf{k}_{\mathbf{p}}$ & $\boldsymbol{\theta}_{\mathbf{p}}$ & $\mathbf{y}\left(\mathbf{t}_{\mathbf{0 . 6 3 2}}\right)$ & $\mathbf{\tau}_{\mathbf{p}}$ & $\mathbf{G}_{\mathbf{i j}}^{\mathbf{a p p r o x}}$ \\
\hline $\mathbf{G 1 1}$ & 0.162 & 1 & 0.162 & 201 & 0.1023 & 4711 & $\frac{0.162 e^{-201 s}}{4510 s+1}$ \\
\hline $\mathbf{G 2 1}$ & - & - & - & - & - & - & 0 \\
\hline $\mathbf{G 2 1}$ & 0.0272 & 1 & 0.0272 & 605 & 0.0172 & 9845 & $\frac{0.0272 e^{-605 s}}{9845 s+1}$ \\
\hline $\mathbf{G 2 2}$ & 0.045 & 1 & 0.045 & 60 & 0.02844 & 440 & $\frac{0.045 e^{-60 s}}{440 s+1}$ \\
\hline
\end{tabular}


Fig. 3, Fig. 4 and Fig. 5 shows the different step responses of the continuous third order functions and their approximations First Order Plus Dead Times of this system which is the infant incubator in open loop. Two curves which are very close to each other are obtained; this is why have been zoomed on the figures to distinguish between the two curves.

As a result of those steps the multivariable system of infant incubator with two inputs and two outputs can be represented as follows:

$\left[\begin{array}{l}y_{1}(s) \\ y_{2}(s)\end{array}\right]=\left[\begin{array}{ll}G_{11}^{\text {approx }} & G_{12}^{\text {approx }} \\ G_{21}^{\text {approx }} & G_{22}^{\text {approx }}\end{array}\right]\left[\begin{array}{l}u_{1}(s) \\ u_{2}(s)\end{array}\right]=\left[\begin{array}{cc}\frac{0.162 e^{-201 s}}{4510 s+1} & 0 \\ \frac{0.0272 e^{-605 s}}{9845 s+1} & \frac{0.045 e^{-60 s}}{440 s+1}\end{array}\right]\left[\begin{array}{l}u_{1}(s) \\ u_{2}(s)\end{array}\right]$

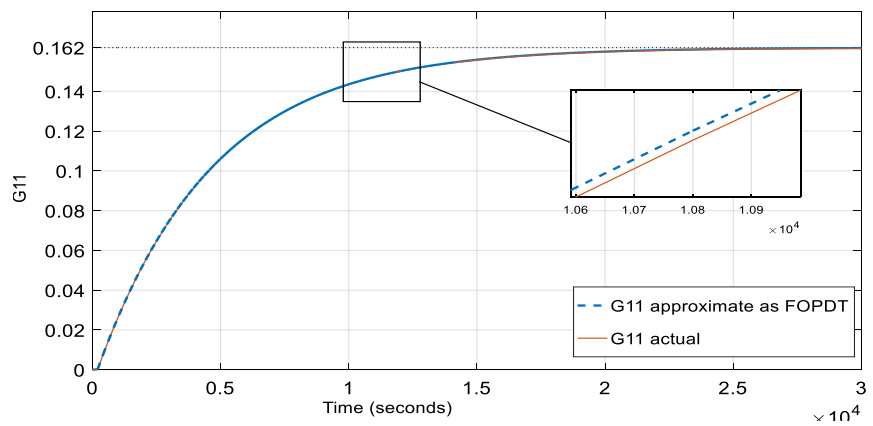

Fig. 3. Comparison of the Open Loop of Step Response between $\mathbf{G}_{11 \text { actual }}(\mathbf{s})$ and $\mathbf{G}_{11 \text { FOPDT }}(\mathbf{s})$.

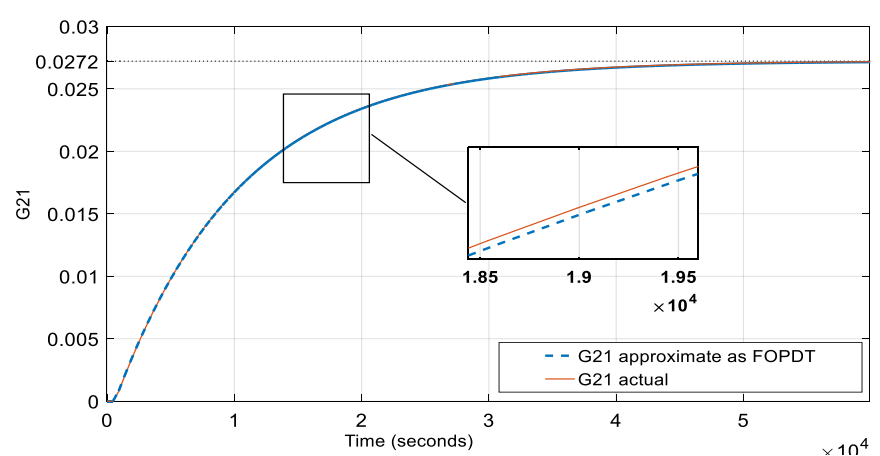

Fig. 4. Comparison of the Open Loop of Step Response between $\mathbf{G}_{21 \text { actual }}(\mathbf{s})$ and $\mathbf{G}_{\text {21FOPDT }}(\mathbf{s})$.

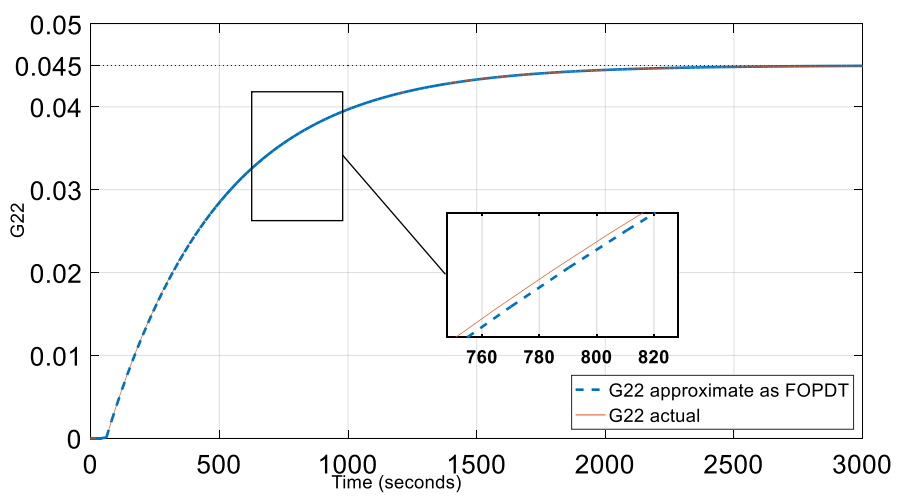

Fig. 5. Comparison of the Open Loop of Step Response between $\mathbf{G}_{22 \text { actual }}(\mathbf{s})$ and $\mathbf{G}_{22 \text { FOPDT }}(\mathbf{s})$.
In equation (12) $y_{1}(s)$ and $y_{2}(s)$ are the process responses and $\mathrm{u}_{1}(\mathrm{~s})$ and $\mathrm{u}_{2}(\mathrm{~s})$ are the inputs functions. Then, the transfer functions $G_{11}^{\text {approx }}, G_{12}^{\text {approx }}, G_{21}^{\text {approx }}$ and $G_{22}^{\text {approx }}$ are taken from Table I. The obtained environmental conditions have been investigated to understand the dynamic relation between the temperature and humidity inside the infant incubator.

To realize the process of testing, the regulation is affected in order to tune the Dynamic Matrix Control. The control horizon of fixed for both temperature and humidity such that Ncu1=Ncu2 $=6$. Concerning the prediction horizon, it is kept to be equal to the maximum lengths of the model which are Npy1 $=1437$ and Npy2=2725. The other parameters are summarized in the Table II. Several changes are made for each figure to prove the varying effect on the tuning of the DMC controller.

TABLE II. TUNING PARAMETERS OF DMC FOR SimUlation OF INFANTINCUBATOR

\begin{tabular}{|c|c|c|c|c|c|}
\hline \multirow[b]{2}{*}{ Case } & \multicolumn{2}{|c|}{ Error weight } & \multicolumn{2}{|c|}{ Control weight } & \multirow{2}{*}{ Figure } \\
\hline & $\alpha_{1}$ & $\alpha_{2}$ & $\lambda_{1}$ & $\lambda_{2}$ & \\
\hline 11 & 6.2 & 1 & 0.035 & 0.052 & \multirow{5}{*}{ Fig. 6} \\
\hline 21 & 6.2 & 5 & 0.035 & 0.052 & \\
\hline 31 & 6.2 & 10 & 0.035 & 0.052 & \\
\hline 41 & 6.2 & 20 & 0.035 & 0.052 & \\
\hline 51 & 6.2 & 30 & 0.035 & 0.052 & \\
\hline 12 & 10.2 & 5 & 0.035 & 0.052 & \multirow{5}{*}{ Fig. 7} \\
\hline 22 & 8.2 & 5 & 0.035 & 0.052 & \\
\hline 32 & 6.2 & 5 & 0.035 & 0.052 & \\
\hline 42 & 10.2 & 5 & 0.035 & 0.052 & \\
\hline 52 & 0.2 & 5 & 0.035 & 0.052 & \\
\hline 13 & 6.2 & 5 & 0.2 & 0.052 & \multirow{5}{*}{ Fig. 8} \\
\hline 23 & 6.2 & 5 & 0.07 & 0.052 & \\
\hline 33 & 6.2 & 5 & 0.05 & 0.052 & \\
\hline 43 & 6.2 & 5 & 0.035 & 0.052 & \\
\hline 53 & 6.2 & 5 & 0.01 & 0.052 & \\
\hline 14 & 6.2 & 5 & 0.035 & 0.12 & \multirow{5}{*}{ Fig. 9} \\
\hline 24 & 6.2 & 5 & 0.035 & 0.07 & \\
\hline 34 & 6.2 & 5 & 0.035 & 0.052 & \\
\hline 44 & 6.2 & 5 & 0.035 & 0.03 & \\
\hline 54 & 6.2 & 5 & 0.035 & 0.01 & \\
\hline
\end{tabular}

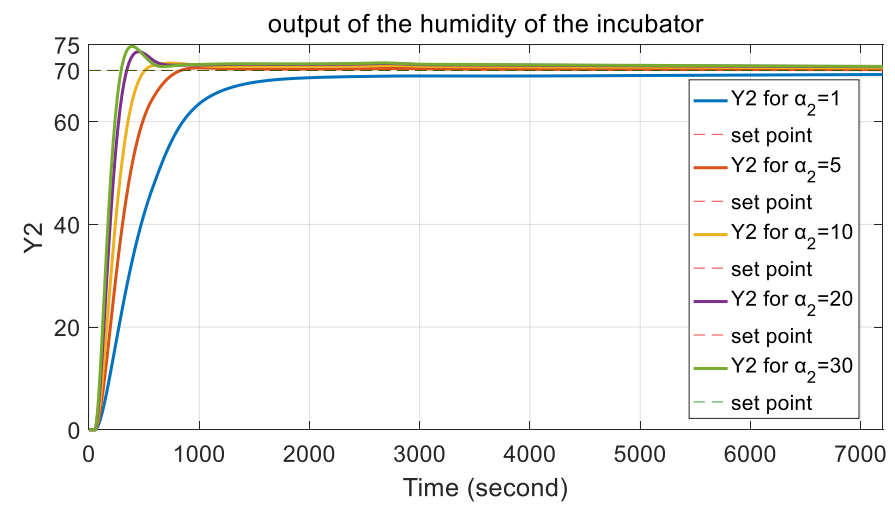

Fig. 6. The Effect of the Error Weight $\left(\boldsymbol{\alpha}_{2}\right)$ on the Humidity. 


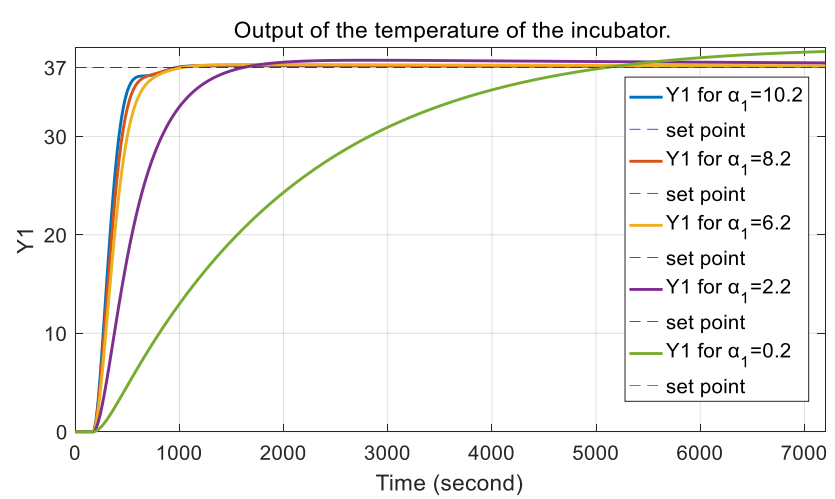

Fig. 7. The Effect of the Control Weight $\left(\boldsymbol{\alpha}_{1}\right)$ on the Temperature.

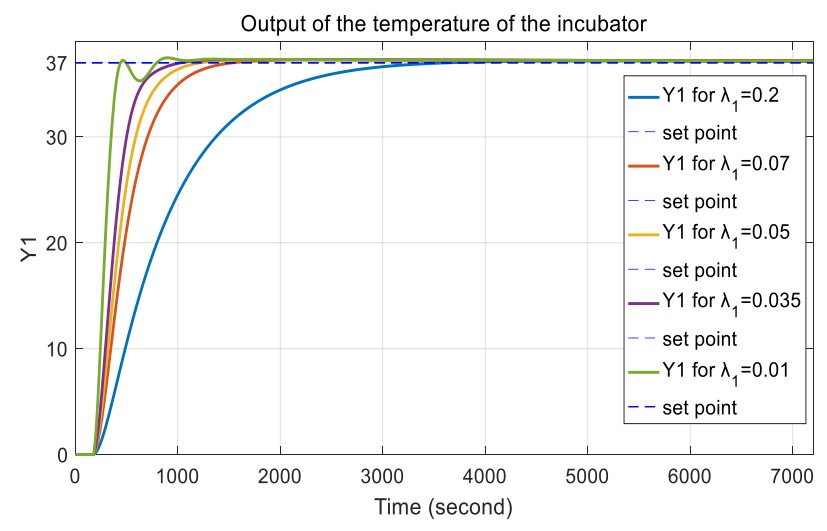

Fig. 8. The Effect of the Control Weight $\left(\boldsymbol{\lambda}_{\mathbf{1}}\right)$ on the Temperature.

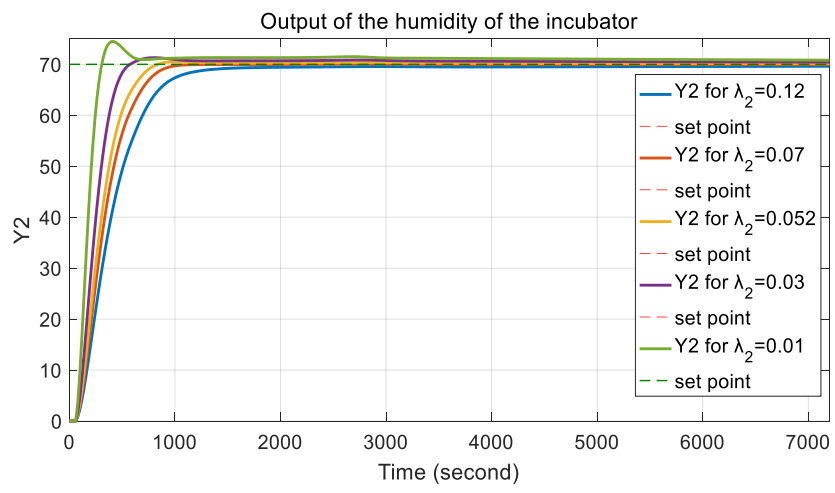

Fig. 9. The Effect of the Control Weight $\left(\boldsymbol{\lambda}_{2}\right)$ on the Humidity.

The effect of the tuning parameters of the infant incubator is illustrated in Fig. 6 through 9 which demonstrate several trials created to tune the Dynamic Matrix Control arranged to achieve the most perfect desirable set of tuning parameters listed in the Table II.

In this paper, spotlighting the effect of the role of the weighting tuning parameters $\alpha_{i}$ and $\lambda_{i}$ which are, the error weight vector and the weight of control move vector, respectively.

Proceeding the trails from the small value of the error weight $\alpha_{2}$ that varies from 1 to 30 . The other parameters starting from case 11 to case 51 are fixed and correspond to Fig. 6, as illustrated in Table II. It is noted that raising the possibility of error makes the responses of the humidity rapid.
Fig. 7 corresponds to the tuning effect of $\alpha_{2}$ which varies from 10.2 to 0.2 . The other fixed values from case 12 to 52 are maintained, as provided in Table II. It is figure out, that the decrease in the error obliges the response of the temperature to be faster in controlling the temperature inside the infant incubator.

Then, Fig. 8 illustrates the tuning effect of the control weight $\lambda_{1}$ which varies from 0.2 to 0.01 . As for the other parameters, they are also fixed. Fig. 8 also shows the result of the change in this parameter, so it is clear that dropping these parameters drive the system to be quick, but with an overshoot equal to 0.8118 .

Finally, Fig. 9 presents the effect of the tuning control weight $\lambda_{2}$ on the humidity which varies from 0.12 to 0.01 , the other values starting from case14 to 54 are fixed, as it is shown in Table II. As the graph demonstrates, when this weight of controlling vector raises, the response of the system is damaged, which makes the response sluggish.

\section{B. Case2: Dynamic Matrix Control for Hito Identification Hidden}

In this part, will focus on the implementation of DMC that was identified by Hito Identification (Herramienta Integrada para Total Optimizacion, Integrated Tool for Total Optimisation) is a software tool oriented to implement MPC control called Hidden toolbox [23] which are a functions are extracted from the previous work. The transfer function of the infant incubator by this software tool under Matlab/environment [23] can be written with a sample time $\mathrm{Ts}=20$ (second), as follows:

$$
\left\{\begin{array}{l}
G_{11}^{\text {hiden }}(z)=z^{-d_{11}} \frac{B_{11}(z)}{A_{11}(z)}=z^{-10} \frac{-0.00010265 z^{-1}+0.00014208 z^{-2}}{1-1.944 z^{-1}+0.9441 z^{-2}} \\
G_{12}^{\text {hiden }}(z)=z^{-d_{12}} \frac{B_{12}(z)}{A_{12}(z)}=0 \\
G_{21}^{\text {hiden }}(z)=z^{-d_{21}} \frac{B_{21}(z)}{A_{21}(z)}=z^{-32} \frac{0.0001286 z^{-1}-0.00011951 z^{-2}}{1-1.9776 z^{-1}+0.977651 z^{-2}} \\
G_{22}^{\text {hiden }}(z)=z^{-d_{22}} \frac{B_{22}(z)}{A_{22}(z)}=z^{-3} \frac{0.0055284 z^{-1}-0.003159 z^{-2}}{1-1.06023 z^{-1}+0.34497 z^{-2}}
\end{array}\right.
$$

Besides, it can to be transformed as a continuous form as follows:

$$
\left\{\begin{array}{l}
G_{11}^{\text {hiden }}(s)=\exp (-200 * s) \frac{-6.306 e-06 s+1.014 e-07}{s^{2}+0.002876 s+2.573 e-07} \\
G_{12}^{\text {hiden }}(s)=0 \\
G_{21}^{\text {hiden }}(s)=\exp (-640 * s) \frac{6.272 e-06 s+2.288 e-08}{s^{2}+0.00113 s+1.264 e-07} \\
G_{22}^{\text {hiden }}(s)=\exp (-60 * s) \frac{0.0003638 s+1.001 e-05}{s^{2}+0.05321 s+0.001203}
\end{array}\right.
$$

Table III below exhibits all the parameters of each response by using the same approximation as method First Order Plus Dead Times as the previous section in paragraph A to obtain the responses of the approximate functions of the system $G_{i j}^{\text {approx }}$.

Fig. 10 to Fig. 12 represents the various step responses of the continuous third order function and their approximations FOPDTs of this system modeling by Hidden identification toolbox under Matlab/environment [23]. 
TABLE III. APPROXIMATION COMPUTED VALUES OF AS FOPDT

\begin{tabular}{|l|l|l|l|l|l|l|l|}
\hline & $\Delta \mathbf{y}$ & $\begin{array}{l}\Delta \\
\mathbf{u}\end{array}$ & $\mathbf{k}_{\mathbf{p}}$ & $\boldsymbol{\theta}_{\mathbf{p}}$ & $\mathbf{y}\left(\mathbf{t}_{\mathbf{0 . 6 3 2}}\right)$ & $\mathbf{\tau}_{\mathbf{p}}$ & $\mathbf{G}_{\mathbf{i j}}^{\mathbf{a p p r o x}}$ \\
\hline $\boldsymbol{G}_{\mathbf{1 1}}^{\text {hiden }}$ & 0.394 & 1 & 0. & $\begin{array}{l}52 \\
0\end{array}$ & 0.249 & $\begin{array}{l}1090 \\
0\end{array}$ & $\frac{0.394 e^{-520 s}}{10900 s+1}$ \\
\hline $\boldsymbol{G}_{\mathbf{1 2}}^{\text {hiden }}$ & - & - & - & - & - & - & 0 \\
\hline $\boldsymbol{G}_{\mathbf{2 1}}^{\text {hiden }}$ & 0.182 & 1 & 0.182 & $\begin{array}{l}97 \\
6\end{array}$ & 0.115 & 8424 & $\frac{0.182 e^{-976 s}}{8424 s+1}$ \\
\hline $\boldsymbol{G}_{22}^{\text {hiden }}$ & 0.008 & 1 & $\begin{array}{l}0.008 \\
3\end{array}$ & 60 & 0.00525 & 19 & $\frac{0.00832 e^{-60 s}}{19 s+1}$ \\
\hline
\end{tabular}

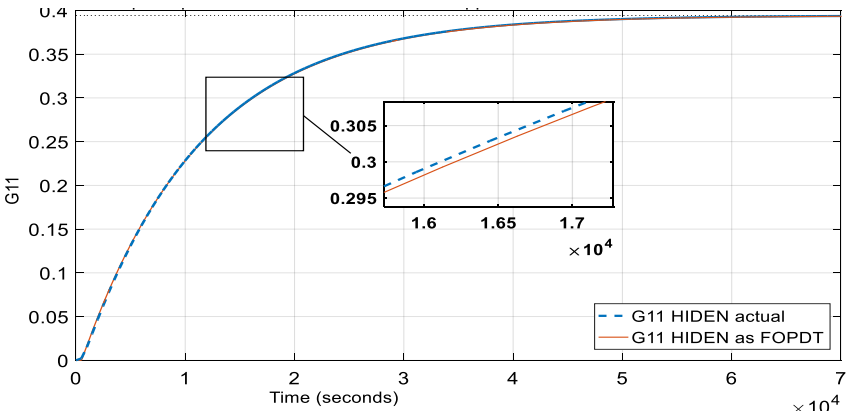

Fig. 10. Comparison of the Open Loop of Step Response between $\mathbf{G}_{11 \text { actual }}^{\text {HIDEN }}(\mathbf{s})$ and $\mathbf{G}_{11 \text { FOPDT }}^{\text {HIDEN }}(\mathbf{s})$.

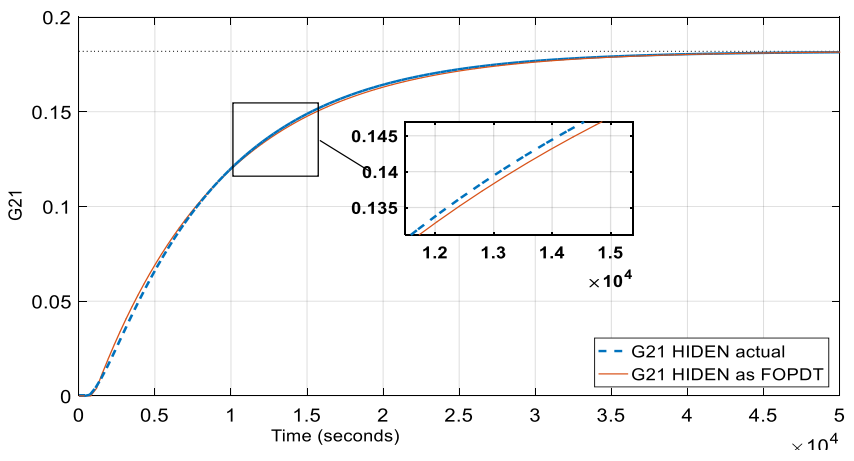

Fig. 11. Comparison of the Open Loop of Step Response between $\mathrm{G}_{21 \text { actual }}^{\mathrm{HIDEN}}(\mathrm{s})$ and $\mathrm{G}_{21 \mathrm{FOPDT}}^{\mathrm{HIDEN}}(\mathrm{s})$.

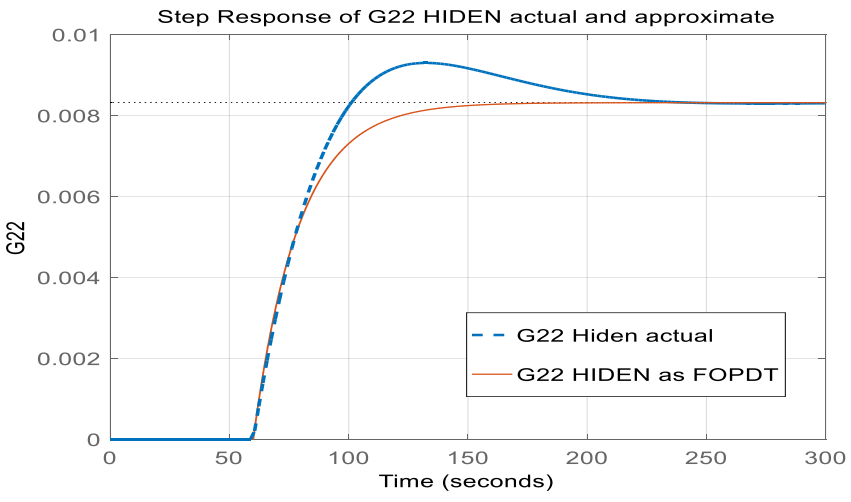

Fig. 12. Comparison of the Open Loop of Step Response between $\mathbf{G}_{22 \text { actual }}^{\text {HIDEN }}(\mathbf{s})$ and $\mathbf{G}_{22 \mathrm{FOPDT}}^{\mathrm{HIDEN}}(\mathbf{s})$.
Table IV contains the parameters of tuning for the DMC controller.

To realize the process of testing, the regulation is affected in order to tune the Dynamic Matrix Control. The control horizon is fixed for both temperature and humidity to be equal to $\mathrm{Ncu} 1=\mathrm{Ncu} 2=6$. The prediction horizon is chosen to be equal to the maximum lengths of the model, namely Npy1=1437 and Npy $2=2725$. The other parameters are summarized in Table IV. Several changed are caused for each figure to prove the varying effect on the tuning of the DMC controller.

TABLE IV. TUNING PARAMETERS OF DMC FOR SiMULATION OF INFANTINCUBATOR

\begin{tabular}{|c|c|c|c|c|c|}
\hline \multirow[b]{2}{*}{ Case } & \multicolumn{2}{|c|}{ Error weight } & \multicolumn{2}{|c|}{ Control weight } & \multirow{2}{*}{ Figure } \\
\hline & $\alpha_{1}$ & $\alpha_{2}$ & $\lambda_{1}$ & $\lambda_{2}$ & \\
\hline 11 & 25 & 10 & 0.3 & 0 & \multirow{5}{*}{ Fig. 13} \\
\hline 21 & 25 & 5 & 0.3 & 0 & \\
\hline 31 & 25 & 0.9 & 0.3 & 0 & \\
\hline 41 & 25 & 0.5 & 0.3 & 0 & \\
\hline 51 & 25 & 0.05 & 0.3 & 0 & \\
\hline 12 & 5 & 2 & 0.3 & 0 & \multirow{5}{*}{ Fig. 14} \\
\hline 22 & 15 & 2 & 0.3 & 0 & \\
\hline 32 & 25 & 2 & 0.3 & 0 & \\
\hline 42 & 35 & 2 & 0.3 & 0 & \\
\hline 52 & 45 & 2 & 0.3 & 0 & \\
\hline 13 & 25 & 2 & 0.09 & 0 & \multirow{5}{*}{ Fig. 15} \\
\hline 23 & 25 & 2 & 0.3 & 0 & \\
\hline 33 & 25 & 2 & 0.8 & 0 & \\
\hline 43 & 25 & 2 & 1.5 & 0 & \\
\hline 53 & 25 & 2 & 2.5 & 0 & \\
\hline 14 & 25 & 2 & 0.3 & $\mathbf{0}$ & \multirow{5}{*}{ Fig. 16} \\
\hline 24 & 25 & 2 & 0.3 & 0.0001 & \\
\hline 34 & 25 & 2 & 0.3 & 0.0009 & \\
\hline 44 & 25 & 2 & 0.3 & 0.01 & \\
\hline 54 & 25 & 2 & 0.3 & 0.1 & \\
\hline
\end{tabular}

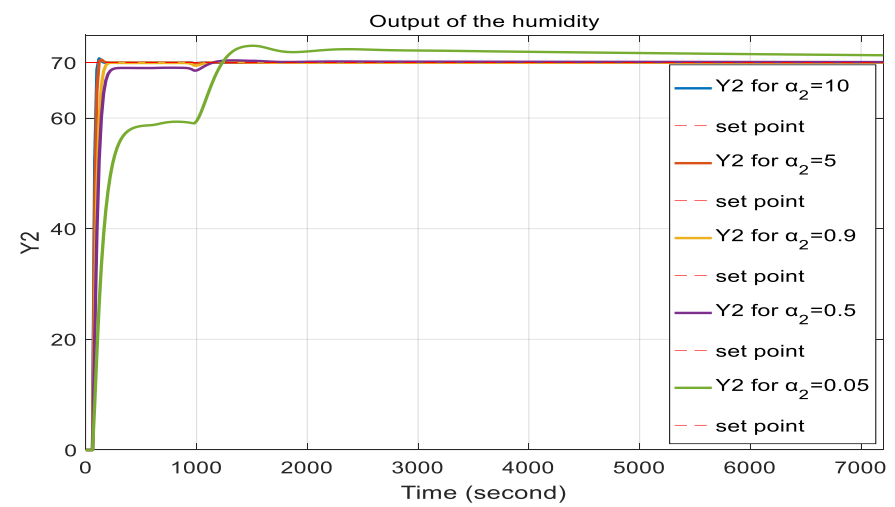

Fig. 13. The Effect of the Control Weight $\left(\alpha_{2}\right)$ on the Humidity. 


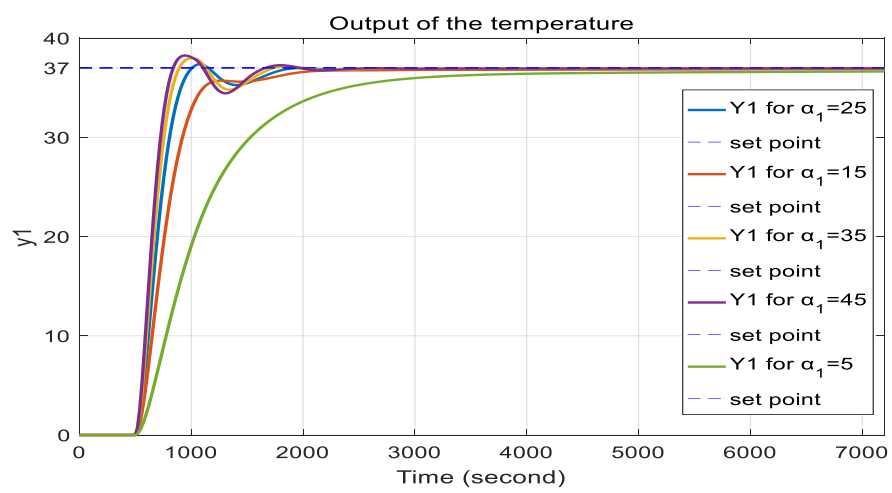

Fig. 14. The Effect of the Control Weight $\left(\boldsymbol{\alpha}_{1}\right)$ on the Temperature.

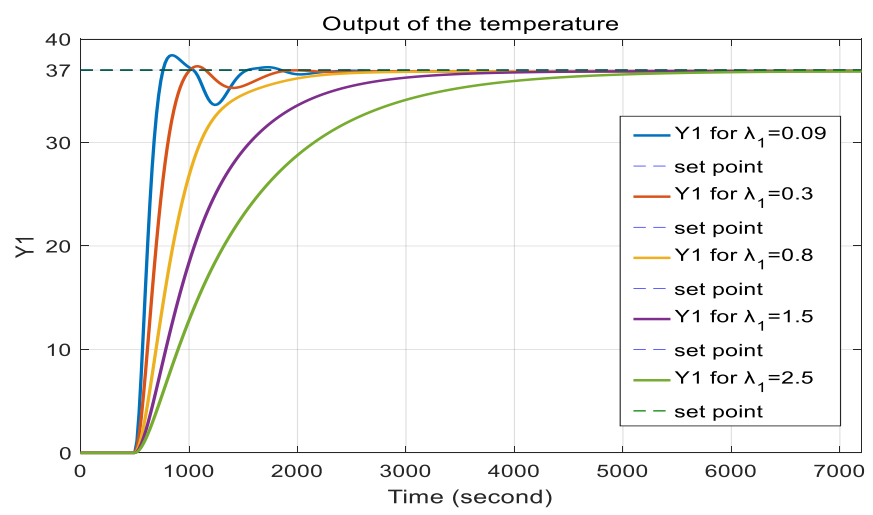

Fig. 15. The Effect of the Smoothing Factor $\left(\boldsymbol{\lambda}_{\mathbf{1}}\right)$ on the Temperature.

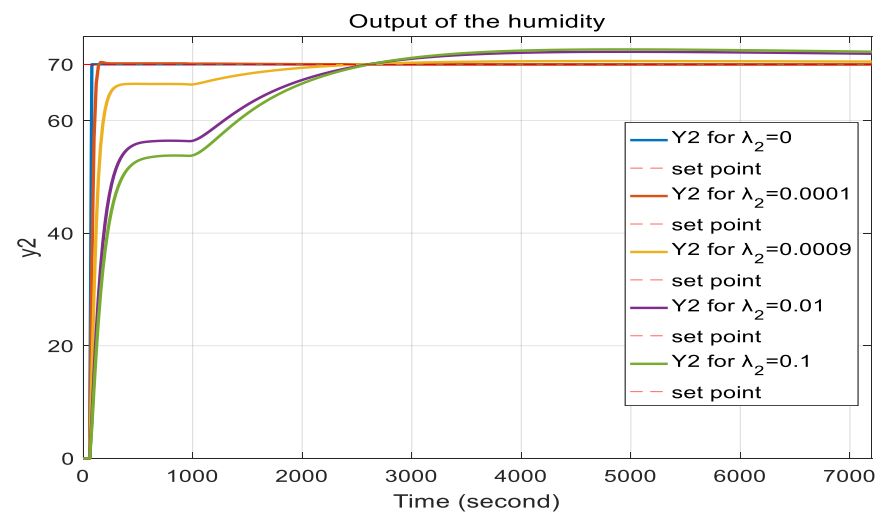

Fig. 16. The Effect of the Smoothing Factor $\left(\boldsymbol{\lambda}_{\mathbf{1}}\right)$ on the Humidity.

The effect of the tuning parameters of the infant incubator is demonstrated in Fig. 13 through 16 which demonstrate the various trials that made it tune the Dynamic Matrix Control arranged to achieve a good set of tuning parameters. These several values are listed in Table IV.

In this part, will be concentrate as the previous part on the effect of the role of the weighting tuning parameters which account for the error weight vector and the weight of control move vector known as $\propto_{i}$ and $\lambda_{i}$, respectively.

The trails begin from an enormous value of the error weight $\propto_{2}$ that varies from 10 to 0.05 . The other parameters starting from case 11 to case 51 are still fixed as it is illustrated in Table IV and correspond to Fig. 13. As previously, noted, the diminution of the error makes the responses of the humidity more unstable and less rapid and lazy.

Fig. 14 coincides with the tuning effect of $\propto_{2}$ varying from 25 to 54 . The other values from case 12 to 52 are settled, as shown in Table IV. It is very noticeable that the reduction in the error obliges the response of the temperature to be faster in controlling the temperature inside the infant incubator. But with this gain in the speed of the peak time, it is very clear that there is an overshot and undershot which are penalizing the system.

Fig. 15 depicts the tuning effect of the control weight $\lambda_{1}$ which fluctuates from 0.09 to 2.5 while the other parameters are fixed. The figure shows the result of the change in this parameter, where it is obvious that dropping these parameters drive the system to be very slow and achieve stability after an interval of time.

Finally, Fig. 16 corresponds to the effect of the tuning control weight $\lambda_{2}$ on the humidity which varies from 0 to 0.1 while the other values from case14 to 54 are fixed as it is presented in Table IV. As previously noticed from the graph, when we increase this weight of controlling vector, the response of the system is penalized and becomes sluggish and lazy.

\section{COMPARATIVE STUDY AND DISCUSSION}

From the curve of the response step which is implemented by Matlab/environment, it is possible to realize different parameters. But, we will only focus on the overshoot and the rise time which is cited in Table $\mathrm{V}$.

Besides, Ziegler-Nichols [27-29] is the researcher who suggested the trial and error method based on sustained oscillations. To obtain the criteria of the curves (response process), there are many methods used to compare the difference between those responses. However, the most popular one is the Integral of the Absolute Error (IAE) which is written as follows:

$$
I A E=\int_{0}^{\infty}|e(t)| . d t
$$

The second method is the Integral of the Square Error (ISE) which is expressed as follows:

$$
I S E=\int_{0}^{\infty} e^{2}(t) \cdot d t
$$

With e $(\mathrm{t})$ is the difference or deviation (error) between the response and the desired set point.

The main goal of the control of the infant incubator application is to maintain the humidity and the temperature at the desired value. Furthermore, the sample time is set to be equal to $20 \mathrm{sec}$. Hence, the parameters of the DMC controller are taken from both Table II and Table IV and given in Table V. Choosing the best performance and smoothest control signal of the output response of both methods for the sake of comparison. 
TABLE V. THE PERFORMANCE COMPARISON OF DMC OF THE INDEX OF THE INCUBATOR

\begin{tabular}{|l|l|l|l|l|}
\hline & \multicolumn{2}{|l|}{ Ident toolbox } & \multicolumn{2}{l|}{ Hidden toolbox } \\
\hline Output response & $\mathbf{T}\left({ }^{\circ} \mathbf{C}\right)$ & $\mathbf{H}(\%)$ & $\mathbf{T}\left({ }^{\circ} \mathbf{C}\right)$ & $\mathbf{H}(\%)$ \\
\hline Overshoot(s) & 0.3872 & 0.4937 & 0 & $1.2503 \mathrm{e}-6$ \\
\hline Rise Time(s) & 17.35 & 21.0045 & 15.847 & 0.8 \\
\hline IAE & 818.3214 & $1.18 \mathrm{e}+3$ & 887.9422 & 280 \\
\hline ISE & $2.2337 \mathrm{e}+4$ & $5.557 \mathrm{e}+4$ & $2.1783 \mathrm{e}+4$ & $2.1791 \mathrm{e}+4$ \\
\hline
\end{tabular}

In this paper, the result is obtained by comparing the time domain specifications and the performance of the index criteria IAE and ISE of both methods which were illustrated in the previous paragraph. In addition, we remove (taken) the peak overshoot and the rise time from the time specifications. The controller which has less error, a minimum rise time and a peak overshoot is reckoned as the best controller. While comparing the time specifications, it is noted that the second method proves a minimum overshoot and less rise time compared to the first method. Moreover, for the criteria index, it is clear the presence of a satisfactory performance of the temperature response, yet an improved performance of the humidity response. Depending on the result of the system that is illustrated in the table below, it is very obvious that the second method of Hidden identification shows an amelioration of the responses of the humidity and the temperature.

Finally, the limitation of this study is presented because it is focus only on the mathematical model of the infant incubator which can be used for the simulation in computer for the infant incubator.

\section{CONCLUSION AND FUTURE WORK}

Different tools and software have been used in this work. The identification is performed on Matlab thanks to two toolbox methods, namely, the ident toolbox and HIDEN. In this paper, all the results of simulation implemented by the Matlab environment with the Dynamic Matrix Control (DMC) controller indicate that the use of this controller leads to good performance. The comparative study made between the two identification tools of the same system allows us to conclude that the identification has a huge effect on the final result that is able to ameliorate the performance, which is an important advantage. In addition, the choice of Dynamic Matrix Control as a controller is due to the ease and efficiency of this strategy. The future work may concern the parameters optimization of the infant incubator with intelligent methods, such as the Particle Swarm Optimization (PSO) and Genetic Algorithm (GA).

\section{REFERENCES}

[1] C. R. Cutler, "Dynamic matrix control: an optimal multivariable control algorithm with constraints," Ph.D. Dissertation, University of Houston, TX,1983.

[2] J. L. Marchetti, A. Duncan, and D. E. Seborg. "Predictive control based on discrete convolution models," Industrial \& Engineering Chemistry Process Design and Development vol. 22( 3), 1983. pp. 488-495.
[3] D. M. Prett and R. D. Gillette, "Optimization and constrained multivariable control of a catalytic cracking unit," Joint automatic control conference. No. 17. 1980.

[4] C. Prada, J. Serrano, P. Vega and M.A. Piera, "A comparative study of GPC and DMC controllers," in Advances in Model Based Predictive Control (D. W. Clarke, Ed.). Oxford University Press, 1994, pp. 38-52.

[5] G. H.C. Oliveira, M. F. Amorim, and C. Pacholok. "A real-time predictive scheme for controlling hygrothermal conditions of neonate incubators," In Proc. of the IFAC Symposium on Modelling and Control of Biomedical Systems, Reims/France, 2006.

[6] G. H.C. Oliveira and Luis. H. Ushijima, "A Hybrid Predictive Control Scheme for Hygrothermal Process," Proceedings of the 17th World Congress, The International Federation of Automatic Control, Seoul, Korea, July, 2008, pp. 6-11,

[7] M. U. Cavalcante, B. C. Torrico, O. da Mota Almeida, A. P. de Souza Brag and F. L. M da Costa Filho, "Filtered model-based predictive control applied to the temperature and humidity control of a neonatal incubator," 2010 9th IEEE/IAS International Conference on Industry Applications-INDUSCON 2010. IEEE, 2010.

[8] M. A. Zermani, E. Feki, A. Mami, "Application of Adaptive Predictive Control to a Newborn Incubator," American J. of Engineering and Applied Sciences, vol. 4(2), 2011, pp. 235-243.

[9] M. A. Zermani, E. Feki and A. Mami, "Application of Genetic Algorithms in identification and control of a new system humidification inside a newborn incubator," International Conference on Communications, Computing and Control Applications, 2011, pp. 1-6.

[10] M. A. Zermani, E. Feki and A. Mami, "Building simulation model of infant-incubator system with decoupling predictive control," IRBM 35, 2014, pp. 189-201.

[11] E. Feki, M. A. Zermani and A. Mami, "GPC temperature control of simulation model infant-incubator and practice with arduino board," IJACSA vol. 8(6), 2017.

[12] C. Ionescu,"Automatic tuning of MPC from step response approximation with FODT and closed loop sensitivity (internal report)," Ghent University, Belgium, 2017.

[13] Q. Bi, W. Cai, L. Lee, Q. G. Wang, C. Hang and Y. Zhang, "Robust identification of first-order plus dead-time model from step response," Control Engineering Practice, vol. 7(1), 1999, pp. 71-77.

[14] D. M. Prett and C. E. García, "Fundamental process control: Butterworths series in chemical engineering," Butterworth-Heinemann. 2013.

[15] R. Shridhar, and J. C. Douglas. "A tuning strategy for unconstrained SISO model predictive control," Industrial \& Engineering Chemistry Research vol. 36(3), 1997, pp. 729-746.

[16] B. W. Bequette, "Process control: modeling, design, and simulation," Prentice Hall Professional, 2003.

[17] J. S Qin and T. A. Badgwell, "An Overview of Industrial Model Predictive Control Technology," Preprints, Proc. Chem. Process Cont. Con\$, CPC V; Tahoe City, California, 1996.

[18] G. M. de Almeida, et al. "Optimal tuning parameters of the dynamic matrix predictive controller with ant colony optimization," 2014 11th IEEE/IAS International Conference on Industry Applications. IEEE, 2014.

[19] P. Bagheri and A. Khaki-Sedigh, "Tuning of dynamic matrix controller for FOPDT models using analysis of variance," IFAC Proceedings Vol. 44(1), 2011, pp. 12319-12324.

[20] D. Dougherty and J. C. Douglas,"Tuning guidelines of a dynamic matrix controller for integrating (non-self-regulating) processes," Industrial \& engineering chemistry research vol. 42(8), 2003, pp. 1739-1752.

[21] T. Klopot, P. Skupin, M. Metzger and P. Grelewicz, "Tuning strategy for dynamic matrix control with reduced horizons," ISA transactions, vol. 76, 2018, pp. 145-154. 
[22] D. M. Prett and R. D. Gillette, "Optimization and constrained multivariable control of a catalytic cracking unit," In Proceedings of the joint automatic control conference, 1980.

[23] J. El Hadj Ali, E. Feki, M. A. Zermani, C. de Prada and A. Mami "Incubator system identification of humidity and temperature: Comparison between two identification environments," $20189^{\text {th }}$ International Renewable Energy Congress (IREC). IEEE, 2018, pp. 1-6.

[24] L. M. Kunene, "Autotuning Predictive Control for Industrial Applications," 2017.

[25] K. Burn, and C. Chris., "Curve fitting software for first order plus dead time (FOPDT) model parameter estimation using step or pulse response data: a tutorial,".
[26] H-P. Huang, and J-C. Jeng., "Process reaction curve and relay methods identification and pid tuning," PID Control. Springer, London, 2005, pp 297-337.

[27] K. Krishnan, and G. Karpagam. "Comparison of PID controller tuning techniques for a FOPDT system," International Journal of Current Engineering and Technology Vol. 4(4) 2667-2670. 2014.

[28] A. Bemporad, M. Morari, and N. Lawrence Ricker. "Model Predictive Control Toolbox User's Guide.” The mathworks. 2010.

[29] K. M. Hussain, R. A. R. Zepherin and M. S. Kumar, "Comparison of tuning methods of PID controllers for FOPTD system," International journal of innovative research in electrical, electronics, instrumentation and control engineering vol. 2(3), 2014, pp. 1177-1180. 